\title{
A NOTE ON THE STABILITY AND UNIQUENESS FOR SOLUTIONS TO THE MINIMAL SURFACE SYSTEM
}

\author{
YNG-ING LeE AND Mu-TaO Wang
}

\begin{abstract}
In this note, we show that the solution to the Dirichlet problem for the minimal surface system in any codimension is unique in the space of distance-decreasing maps. This follows as a corollary of the following stability theorem: if a minimal submanifold $\Sigma$ is the graph of a (strictly) distance-decreasing map, then $\Sigma$ is (strictly) stable. It is known that a minimal graph of codimension one is stable without assuming the distance-decreasing condition. We give another criterion for the stability in terms of the two-Jacobians of the map which in particular covers the codimension one case. All theorems are proved in the more general setting for minimal maps between Riemannian manifolds. The complete statements of the results appear in Theorem 3.1, Theorem 3.2, and Theorem 4.1.
\end{abstract}

\section{Introduction}

Let $\Omega$ be a bounded domain in $\mathbb{R}^{n}$. Recall a $C^{2}$ vector-valued function $f=$ $\left(f^{1}, \cdots, f^{m}\right): \Omega \rightarrow \mathbb{R}^{m}$ is said to be a solution to the minimal surface system (see Osserman [OS] or Lawson-Osserman [LO]) if

$$
\sum_{i, j=1}^{n} \frac{\partial}{\partial x^{i}}\left(\sqrt{g} g^{i j} \frac{\partial f^{\alpha}}{\partial x^{j}}\right)=0 \text { for each } \alpha=1 \cdots m
$$

where $g_{i j}=\delta_{i j}+\sum_{\alpha} \frac{\partial f^{\alpha}}{\partial x^{i}} \frac{\partial f^{\alpha}}{\partial x^{j}}, g=\operatorname{det} g_{i j}$ and $g^{i j}$ is the $(i, j)$ entry of the inverse matrix of $\left(g_{i j}\right)$. The graph of $f$ is called a non-parametric minimal submanifold. Equation (1.1) is indeed the Euler-Lagrange equation of the volume functional $\int_{\Omega} \sqrt{g} d x^{1} \wedge \cdots \wedge d x^{n}$.

In the codimension one case, i.e. $m=1$, a simple calculation shows $g^{i j}=\delta_{i j}-$ $\frac{f_{i} f_{j}}{1+|\nabla f|^{2}}$ and the equation is equivalent to the familiar one,

$$
\operatorname{div}\left(\frac{\nabla f}{\sqrt{1+|\nabla f|^{2}}}\right)=0 .
$$

It is well-known that the solution to (1.2) subject to the Dirichlet boundary condition is unique and stable(see for example, Lawson-Osserman [LO]).

However in the higher codimension case $(m>1)$, Lawson and Osserman [LO] discover a remarkable counterexample to the uniqueness and stability of solutions of (1.1) when $n=m=2$. They construct two distinct non-parametric minimal surfaces with the same boundary. Lawson and Osserman then show an unstable non-parametric minimal surface with the same boundary exists as a result of the

Received by the editors March 7, 2007. 
theorems of Morse-Tompkins [MT] and Shiffman [SH]. In the same paper, Lawson and Osserman show the Dirichlet problem for the minimal surface system may not be solvable in higher codimension.

In this paper, we first derive a stability criterion for the minimal surface system in higher codimension. To describe the results, we define distance-decreasing maps.

Definition 1. A map $f: \Omega \subset \mathbb{R}^{n} \rightarrow \mathbb{R}^{m}$ is called distance-decreasing if the differential df satisfies $|d f(v)| \leq|v|$ at each point of $\Omega$ for any nonzero vector $v \in \mathbb{R}^{n}$. It is called strictly distance-decreasing if $|d f(v)|<|v|$ at each point of $\Omega$ for any nonzero vector $v \in \mathbb{R}^{n}$.

We prove the following stability theorem.

Theorem A (see Theorem 3.1) Suppose a nonparametric minimal submanifold $\Sigma$ is the graph of a distance-decreasing map $f: \Omega \subset \mathbb{R}^{n} \rightarrow \mathbb{R}^{m}$. Then $\Sigma$ is stable. It is strictly stable if $f$ is strictly distance-decreasing.

This theorem generalizes the stability criterion in [LW]. It turns out the volume element is a convex function on the space of distance-decreasing linear transformations. The convexity is further exploited to derive a uniqueness criterion. Namely, we show the solution to the Dirichlet problem for the minimal surface system is unique in the space of distance-decreasing maps.

Theorem B (see Theorem 3.2) Suppose that $\Sigma_{0}$ and $\Sigma_{1}$ are nonparametric minimal submanifolds which are the graph of $f_{0}: \Omega \subset \mathbb{R}^{n} \rightarrow \mathbb{R}^{m}$ and $f_{1}: \Omega \subset \mathbb{R}^{n} \rightarrow \mathbb{R}^{m}$ respectively. If both $f_{0}$ and $f_{1}$ are distance-decreasing and $f_{0}=f_{1}$ on $\partial \Omega$, then $\Sigma_{0}=\Sigma_{1}$

We remark that solutions to the Dirichlet problem of minimal surface systems in higher codimensions are constructed in [WA1] and the solutions are graphs of distancedecreasing maps. For earlier uniqueness theorems for minimal surfaces, we refer to Meek's paper [ME].

We prove slightly more general stability and uniqueness theorems for minimal maps between Riemannian manifolds in this paper. It turns out the only extra assumption is on the sign of the curvature of the target manifold. In particular, Theorem 3.1 implies Theorem A while Theorem 3.2 implies Theorem B.

It is well-known that any minimal graph of codimension one is volume-minimizing by a calibration argument. To connect to the codimension one case, we develop another stability criterion for the minimal surface system in any codimension in section 4 . The criterion is in terms of the two-Jacobians of $f$. To describe the results, we first recall some notations. Let $L: \mathbb{R}^{n} \rightarrow \mathbb{R}^{m}$ be a linear transformation. It induces a linear transformation $\wedge^{2} L$, from the wedge product $\wedge^{2} \mathbb{R}^{n}$ to $\wedge^{2} \mathbb{R}^{m}$ by

$$
\left(\wedge^{2} L\right)(v \wedge w)=L(v) \wedge L(w) .
$$

With this we define

$$
\left|\wedge^{2} L\right|=\sup _{|v \wedge w|=1}\left|\left(\wedge^{2} L\right)(v \wedge w)\right| .
$$

In particular, $\left|\wedge^{2} L\right|=0$ if $L$ is of rank one. 
Theorem C (see Theorem 4.1) Suppose a nonparametric minimal submanifold $\Sigma$ is the graph of a map $f: \Omega \subset \mathbb{R}^{n} \rightarrow \mathbb{R}^{m}$. Then $\Sigma$ is stable if $\left|\wedge^{2} d f\right|(x) \leq \frac{1}{n-1}$.

A more refined and more general version is proved in Theorem 4.1. The rank of the defining function $f$ of a nonparametric minimal submanifold of codimension one is at most one and thus $\left|\wedge^{2} d f\right|(x)=0$. We prove the results for minimal maps between Riemannian manifolds as stated in Theorem 4.1.

\section{A non-parametric variation formula for graphs}

Suppose that $(M, g)$ and $(N, h)$ are two Riemannian manifolds with dimension $n$ and $m$ respectively. We fix a local coordinate system $\left\{x^{i}\right\}$ on $M$. Let $f$ be a smooth map from $(M, g)$ to $(N, h)$. The graph of $f$ is an embedded submanifold of the product manifold $M \times N$, the induced metric is given by

$$
\sum_{i, j=1}^{n} G_{i j} d x^{i} d x^{j}=\sum_{i, j=1}^{n}\left(g_{i j}+\left\langle d f\left(\frac{\partial}{\partial x^{i}}\right), d f\left(\frac{\partial}{\partial x^{j}}\right)\right\rangle\right) d x^{i} d x^{j},
$$

and the volume of the graph is

$$
A=\int_{M} \sqrt{\operatorname{det} G_{i j}} d x^{1} \wedge \cdots \wedge d x^{n}=\int_{M} d v
$$

Assume that there is a family of maps $f_{t}, 0 \leq t \leq \epsilon$ from $M$ to $N$ with $f_{0}=f$ on $M$ and $f_{t}=f$ outside a compact subset of $M$. When the boundary of $M$ is nonempty, we require that $f_{t}=f$ on $\partial M$. In the following, we compute the first and second variations of the volumes of the graphs. The variation of the volume form is

$$
\frac{d \sqrt{\operatorname{det} G_{i j}(t)}}{d t}=\frac{1}{2} \sum_{i, j} G^{i j}(t) \dot{G}_{i j}(t) \sqrt{\operatorname{det} G_{i j}(t)},
$$

where $G^{i j}(t)$ is the $(i, j)$ entry of the inverse matrix of $\left(G_{i j}(t)\right)$.

Denote the variation field $\frac{d f_{t}}{d t}$ by $V(t)$. For simplicity, we omit the dependency of $G_{i j}$ and $V$ on $t$ in the following calculation. Then

$$
\begin{aligned}
\dot{G}_{i j} & =\left\langle\nabla_{V} d f_{t}\left(\frac{\partial}{\partial x^{i}}\right), d f_{t}\left(\frac{\partial}{\partial x^{j}}\right)\right\rangle+\left\langle d f_{t}\left(\frac{\partial}{\partial x^{i}}\right), \nabla_{V} d f_{t}\left(\frac{\partial}{\partial x^{j}}\right)\right\rangle \\
& =\left\langle\nabla_{d f_{t}\left(\frac{\partial}{\partial x^{i}}\right)} V, d f_{t}\left(\frac{\partial}{\partial x^{j}}\right)\right\rangle+\left\langle d f_{t}\left(\frac{\partial}{\partial x^{i}}\right), \nabla_{d f_{t}\left(\frac{\partial}{\partial x^{j}}\right)} V\right\rangle .
\end{aligned}
$$

Here $\nabla$ is the Riemannian connection on $N$, and $V$ and $d f_{t}\left(\frac{\partial}{\partial x^{i}}\right)$ are vector fields tangent to $N$.

Hence the first variation formula is

$$
\frac{d A_{t}}{d t}=\int_{M} \sum_{i, j} G^{i j}\left\langle\nabla_{d f_{t}\left(\frac{\partial}{\partial x^{i}}\right)} V, d f_{t}\left(\frac{\partial}{\partial x^{j}}\right)\right\rangle d v_{t} .
$$

Continuing the computation, we derive

$$
\frac{d^{2} A_{t}}{d t^{2}}=\frac{1}{2} \int_{M}\left(\sum_{i, j} G^{i j} \ddot{G}_{i j}-\sum_{i, j, k, l} G^{i k} \dot{G}_{k l} G^{l j} \dot{G}_{i j}\right) d v_{t}+\frac{1}{4} \int_{M}\left(\sum_{i, j} G^{i j} \dot{G}_{i j}\right)^{2} d v_{t}
$$


Now

$$
\begin{aligned}
\ddot{G}_{i j}= & \left\langle\nabla_{V} \nabla_{d f_{t}\left(\frac{\partial}{\partial x^{i}}\right)} V, d f_{t}\left(\frac{\partial}{\partial x^{j}}\right)\right\rangle+\left\langle d f_{t}\left(\frac{\partial}{\partial x^{i}}\right), \nabla_{V} \nabla_{d f_{t}\left(\frac{\partial}{\partial x^{j}}\right)} V\right\rangle \\
& +2\left\langle\nabla_{V} d f_{t}\left(\frac{\partial}{\partial x^{i}}\right), \nabla_{V} d f_{t}\left(\frac{\partial}{\partial x^{j}}\right)\right\rangle \\
= & \left\langle R\left(V, d f_{t}\left(\frac{\partial}{\partial x^{i}}\right)\right) V, d f_{t}\left(\frac{\partial}{\partial x^{j}}\right)\right\rangle+\left\langle\nabla_{d f_{t}\left(\frac{\partial}{\partial x^{i}}\right)} \nabla_{V} V, d f_{t}\left(\frac{\partial}{\partial x^{j}}\right)\right\rangle \\
& +\left\langle R\left(V, d f_{t}\left(\frac{\partial}{\partial x^{j}}\right)\right) V, d f_{t}\left(\frac{\partial}{\partial x^{i}}\right)\right\rangle+\left\langle d f_{t}\left(\frac{\partial}{\partial x^{i}}\right), \nabla_{d f_{t}\left(\frac{\partial}{\partial x^{j}}\right)} \nabla_{V} V\right\rangle \\
& +2\left\langle\nabla_{d f_{t}\left(\frac{\partial}{\partial x^{i}}\right)} V, \nabla_{d f_{t}\left(\frac{\partial}{\partial x^{j}}\right)} V\right\rangle .
\end{aligned}
$$

Symmetrizing the indexes, the second variation formula becomes

$$
\begin{aligned}
\frac{d^{2} A_{t}}{d t^{2}} & =\int_{M}\left(\sum_{i, j} G^{i j}\left\langle\nabla_{d f\left(\frac{\partial}{\partial x^{i}}\right)} V, \nabla_{d f\left(\frac{\partial}{\partial x^{j}}\right)} V\right\rangle-\frac{1}{2} \sum_{i, j, k, l} G^{i k} \dot{G}_{k l} G^{l j} \dot{G}_{i j}\right) d v_{t} \\
& +\int_{M} \sum_{i, j} G^{i j}\left\langle R\left(V, d f\left(\frac{\partial}{\partial x^{j}}\right)\right) V, d f\left(\frac{\partial}{\partial x^{i}}\right)\right\rangle d v_{t}+\frac{1}{4} \int_{M}\left(\sum_{i, j} G^{i j} \dot{G}_{i j}\right)^{2} d v_{t} \\
& +\int_{M} \sum_{i, j} G^{i j}\left\langle\nabla_{d f\left(\frac{\partial}{\partial x^{i}}\right)} \nabla_{V} V, d f\left(\frac{\partial}{\partial x^{j}}\right)\right\rangle d v_{t} .
\end{aligned}
$$

This formula will be used to prove the main theorems in the next section.

\section{The stability and uniqueness of minimal maps}

We recall a minimal submanifold is called stable if the second derivative of the volume functional with respect to any compact supported normal variation is nonnegative. We prove the following lemma for minimal graphs.

Lemma 3.1. Suppose that the graph of $f: M \rightarrow N$ is a minimal submanifold $\Sigma$ in $M \times N$. Then $\Sigma$ is stable if and only if it is stable with respect to any compact supported deformation of maps from $M$ to $N$.

Proof. Suppose that $a_{i}$ is an orthonormal basis of the principal directions of $d f$ with stretches $\lambda_{i} \geq 0$ and that $d f\left(a_{i}\right)=\lambda_{i} b_{i}$. Assume that the rank of $d f(x)$ is $p$. The orthonormal set $\left\{b_{i}\right\}_{i=1 \cdots p}$ can be completed to form a local orthonormal basis $\left\{b_{\alpha}\right\}_{\alpha=1 \cdots m}$ of the tangent space of $N$. In the basis chosen as above, the tangent space of $\Sigma$ is spanned by $t_{i}=\frac{1}{\sqrt{1+\lambda_{i}^{2}}}\left(a_{i}+\lambda_{i} b_{i}\right), \quad 1 \leq i \leq n$. Observe that $\lambda_{i}=0$ for $p<i \leq n$. The normal space of $\Sigma$ is spanned by $n_{i}=\frac{1}{\sqrt{1+\lambda_{i}^{2}}}\left(b_{i}-\lambda_{i} a_{i}\right), \quad 1 \leq i \leq p$ and $n_{\alpha}=b_{\alpha}$ for $p<\alpha \leq m$. Assume that $\bar{V}=\sum_{\alpha=1}^{m} v_{\alpha} n_{\alpha}$ is a compact supported normal vector field along $\Sigma$. Then the compact supported vector field $V=$ $\sum_{i} \sqrt{1+\lambda_{i}^{2}} v_{i} b_{i}+\sum_{\alpha>p} v_{\alpha} b_{\alpha}$ tangent to $N$ satisfies $V^{\perp}=\bar{V}$, where $(\cdot)^{\perp}$ denotes the normal part of a vector, i.e. the projection onto the normal space of $\Sigma$. The second derivative of volume functional in the direction $V^{\perp}=\bar{V}$ is the same as in the direction $V$. The Lemma is thus proved.

The notion of a (strictly) distance-decreasing map in Definition 1 can be generalized to maps between Riemannian manifolds and we can prove the following theorem. 
Theorem 3.1. Suppose that $M$ and $N$ are two Riemannian manifolds with dimension $n$ and $m$ respectively, and the sectional curvature of $N$ is non-positive. Assume that $f: M \rightarrow N$ is a distance-decreasing map and the graph of $f$, which is denoted by $\Sigma$, is minimal in $M \times N$. Then the minimal submanifold $\Sigma$ is stable. It is strictly stable in the following two cases:

(i) $N$ has negative sectional curvature, and $f$ is not a constant map.

(ii) $f$ is strictly distance-decreasing, and $M$ is noncompact or with nonempty boundary.

Proof. On a minimal submanifold, we have $\left.\frac{d A_{t}}{d t}\right|_{t=0}=0$ for any variation field and in particular

$$
\int_{M} \sum_{i, j} G^{i j}\left\langle\nabla_{d f\left(\frac{\partial}{\partial x^{i}}\right)} \nabla_{V} V, d f\left(\frac{\partial}{\partial x^{j}}\right)\right\rangle d v=0 .
$$

In the basis chosen in the proof of Lemma 3.1, we derive from (2.3) the following inequality

$$
\begin{aligned}
\left.\frac{d^{2} A_{t}}{d t^{2}}\right|_{t=0} \geq & \int_{M}\left(\sum_{i} \frac{1}{1+\lambda_{i}^{2}}\left(\left|\nabla_{d f\left(a_{i}\right)} V\right|^{2}-\left\langle R\left(V, d f\left(a_{i}\right)\right) d f\left(a_{i}\right), V\right\rangle\right)\right. \\
& \left.-\frac{1}{2} \sum_{i, j} \frac{1}{1+\lambda_{i}^{2}} \frac{1}{1+\lambda_{j}^{2}}\left(\left\langle\nabla_{d f\left(a_{i}\right)} V, d f\left(a_{j}\right)\right\rangle+\left\langle\nabla_{d f\left(a_{j}\right)} V, d f\left(a_{i}\right)\right\rangle\right)^{2}\right) d v .
\end{aligned}
$$

Since the sectional curvature of $N$ is non-positive, this becomes

$$
\begin{aligned}
\left.\frac{d^{2} A_{t}}{d t^{2}}\right|_{t=0} \geq & \int_{M}\left(\sum_{i} \frac{1}{1+\lambda_{i}^{2}}\left|\nabla_{d f\left(a_{i}\right)} V\right|^{2}\right. \\
& \left.-\frac{1}{2} \sum_{i, j} \frac{1}{1+\lambda_{i}^{2}} \frac{1}{1+\lambda_{j}^{2}}\left(\lambda_{j}\left\langle\nabla_{d f\left(a_{i}\right)} V, b_{j}\right\rangle+\lambda_{i}\left\langle\nabla_{d f\left(a_{j}\right)} V, b_{i}\right\rangle\right)^{2}\right) d v \\
\geq & \int_{M}\left(\sum_{i, j} \frac{1}{1+\lambda_{i}^{2}}\left\langle\nabla_{d f\left(a_{i}\right)} V, b_{j}\right\rangle^{2}\right. \\
& \left.-\sum_{i, j} \frac{1}{1+\lambda_{i}^{2}} \frac{1}{1+\lambda_{j}^{2}}\left(\lambda_{j}^{2}\left\langle\nabla_{d f\left(a_{i}\right)} V, b_{j}\right\rangle^{2}+\lambda_{i}^{2}\left\langle\nabla_{d f\left(a_{j}\right)} V, b_{i}\right\rangle^{2}\right)\right) d v \\
= & \int_{M} \sum_{i, j} \frac{\left\langle\nabla_{d f\left(a_{i}\right)} V, b_{j}\right\rangle^{2}}{1+\lambda_{i}^{2}} \frac{1-\lambda_{j}^{2}}{1+\lambda_{j}^{2}} d v .
\end{aligned}
$$

When $f$ is a distance-decreasing map, we have $\lambda_{j} \leq 1$ for $1 \leq j \leq n$. From the estimate in (3.2), it follows that $\left.\frac{d^{2} A_{t}}{d t^{2}}\right|_{t=0} \geq 0$. This implies that $\Sigma$ is stable by Lemma 3.1. Suppose that $f$ is strictly distance-decreasing, i.e. $\lambda_{j}<1$ for $1 \leq j \leq n$. If $\left.\frac{d^{2} A_{t}}{d t^{2}}\right|_{t=0}=0$, it implies that $\left\langle\nabla_{d f\left(a_{i}\right)} V, b_{j}\right\rangle=0$ for $1 \leq i, j \leq n$ and $\left|\nabla_{d f\left(a_{i}\right)} V\right|^{2}=$ $\sum_{j}\left\langle\nabla_{d f\left(a_{i}\right)} V, b_{j}\right\rangle^{2}$. Hence $\nabla_{d f\left(a_{i}\right)} V=0$ for $1 \leq i \leq n$. That is, $V$ is a parallel vector field. In case (ii), $V$ either vanishes outside a compact set or on the boundary of $M$, so the parallel condition implies that $V$ is a zero vector. This proves that $\Sigma$ is strictly stable in case (ii). When the sectional curvature of $N$ is negative and $f$ is not a constant map, one always has $\left.\frac{d^{2} A_{t}}{d t^{2}}\right|_{t=0}>0$ unless $V$ is a zero vector. Therefore, $\Sigma$ is strictly stable in case (i). 
Remark 1. In case that $M$ is compact without boundary and $f$ is strictly distancedecreasing, one still has the following conclusion: If $\left.\frac{d^{2} A_{t}}{d t^{2}}\right|_{t=0}=0$, then $V$ is a parallel vector field and $\left\langle R\left(V, d f_{0}\left(a_{i}\right)\right) d f_{0}\left(a_{i}\right), V\right\rangle=0$ for $1 \leq i \leq n$.

Using the second variation formula, we can also prove the uniqueness of minimal maps.

Theorem 3.2. Suppose that $M$ and $N$ are two Riemannian manifolds with dimension $n$ and $m$ respectively, and the sectional curvature of $N$ is non-positive. Let $\Sigma_{0}$ and $\Sigma_{1}$ be minimal submanifolds in $M \times N$, which are the graphs of distance-decreasing maps $f_{0}: M \rightarrow N$ and $f_{1}: M \rightarrow N$, respectively. Assume that $f_{0}$ and $f_{1}$ are homotopic, and are identical on the boundary of $M$ and outside a compact set of $M$. Then $\Sigma_{0}=\Sigma_{1}$ in the following two cases:

(i) The sectional curvature of $N$ is negative, and $f_{1}$ and $f_{2}$ are not constant maps,

(ii) The boundary of $M$ is nonempty, or $M$ is noncompact.

Proof. Lift the homotopy map between $f_{0}$ and $f_{1}$ to the universal covering of $N$. Because the sectional curvature of $N$ is non-positive, there exists a unique geodesic connecting the lifting $\widetilde{f}_{0}(x)$ and $\widetilde{f}_{1}(x)$. Denote the projection of this unique geodesic onto $N$ by $\gamma_{x}(t)$ and define $f_{t}(x)=\gamma_{x}(t)$. Then $V=\dot{\gamma}_{x}(t)$ satisfies $\nabla_{V} V=0$. Hence the same bound on $\frac{d^{2} A_{t}}{d t^{2}}$ as in (3.2) holds for $0 \leq t \leq 1$.

The vector field $d f_{t}\left(\frac{\partial}{\partial x^{i}}\right), 0 \leq t \leq 1$, is a Jacobi field along $\gamma_{x}(t)$, which is denoted by $J_{i, x}(t)$. A direct calculation gives

$$
\frac{d^{2}}{d t^{2}}\left|J_{i, x}\right|^{2}=2\left\langle\ddot{J}_{i, x}, J_{i, x}\right\rangle+2\left|\dot{J}_{i, x}\right|^{2}=2\left\langle R\left(V, J_{i, x}\right) V, J_{i, x}\right\rangle+2\left|\dot{J}_{i, x}\right|^{2} \geq 0 .
$$

The last inequality follows from the fact that $N$ has nonpositive sectional curvature. Because both $f_{0}$ and $f_{1}$ are distance-decreasing maps, one has $\left|J_{i, x}(0)\right|^{2} \leq\left|\frac{\partial}{\partial x^{i}}\right|^{2}$ and $\left|J_{i, x}(1)\right|^{2} \leq\left|\frac{\partial}{\partial x^{i}}\right|^{2}$. The inequality (3.3) then implies $\left|J_{i, x}(t)\right|^{2} \leq\left|\frac{\partial}{\partial x^{i}}\right|^{2}$. Hence $f_{t}$ is also distance-decreasing and one concludes that $\frac{d^{2} A_{t}}{d t^{2}} \geq 0$ from (3.2) for $0 \leq t \leq 1$. Because $\left.\frac{d A_{t}}{d t}\right|_{t=0}=\left.\frac{d A_{t}}{d t}\right|_{t=1}=0$, the bound gives $\frac{d A_{t}}{d t}=0$ and $\frac{d^{2} A_{t}}{d t^{2}}=0$ for $0 \leq t \leq 1$. In order to have $\left.\frac{d^{2} A_{t}}{d t^{2}}\right|_{t=0}=0$, every inequality in (3.1) and (3.2) should be achieved by an equality. It implies that the following conditions must hold:

(1) $\sum_{i} \frac{1}{1+\lambda_{i}^{2}}\left\langle\nabla_{d f_{0}\left(a_{i}\right)} V, d f_{0}\left(a_{i}\right)\right\rangle=0$.

(2) $\left|\nabla_{d f_{0}\left(a_{i}\right)} V\right|^{2}=\sum_{j}\left\langle\nabla_{d f_{0}\left(a_{i}\right)} V, b_{j}\right\rangle^{2}$ for $1 \leq i \leq n$.

(3) $\left\langle\nabla_{d f_{0}\left(a_{i}\right)} V, d f_{0}\left(a_{j}\right)\right\rangle=\left\langle\nabla_{d f_{0}\left(a_{j}\right)} V, d f_{0}\left(a_{i}\right)\right\rangle$ for $1 \leq i, j \leq n$.

(4) If $\lambda_{j}<1$, then $\left\langle\nabla_{d f_{0}\left(a_{i}\right)} V, b_{j}\right\rangle=0$ for $1 \leq i \leq n$. It in particular implies $\left\langle\nabla_{d f_{0}\left(a_{i}\right)} V, d f_{0}\left(a_{j}\right)\right\rangle=0$ for $1 \leq i \leq n$.

(5) $\left\langle R\left(V, d f_{0}\left(a_{i}\right)\right) d f_{0}\left(a_{i}\right), V\right\rangle=0$ for $1 \leq i \leq n$.

Condition 1 follows from the fact that we drop $\frac{1}{4} \int_{M}\left(\sum_{i, j} G^{i j} \dot{G}_{i j}\right)^{2} d v_{t}$ in (2.3) to get (3.1). An equality in (3.1) implies $\sum_{i, j} G^{i j} \dot{G}_{i j}=0$ and condition 1 is the same equation in the basis chosen as in the proof of Lemma 3.1. If the first inequality in (3.2) is achieved by an equality, condition 5 must hold. Condition 2 and 3 are necessary to obtain an equality in the second inequality in (3.2). Note that in condition 2 , we only sum over the projections to $b_{j}, 1 \leq j \leq n$, instead of the complete basis $b_{\alpha}$, 
$1 \leq \alpha \leq m$. Finally in the last equality of (3.2), we must have every term vanishing since $\lambda_{j} \leq 1$ for $1 \leq j \leq n$. This gives condition 4 .

Now we use these conditions to prove the theorem. When the sectional curvature of $N$ is negative and $f_{0}$ is not a constant map, condition 5 implies that $V=0$. Hence $f_{0}=f_{1}$ and $\Sigma_{0}=\Sigma_{1}$. It shows case (i).

Suppose that the sectional curvature of $N$ is non-positive. We will show that $V$ is a parallel vector field. That is, $\nabla_{d f_{0}\left(a_{j}\right)} V=0$ for any $1 \leq j \leq n$. Fix a point $x \in M$ and choose coordinates at $x$ such that $a_{j}=\frac{\partial}{\partial x^{j}}$ for $1 \leq j \leq n$. If $\lambda_{j}=1$, we have $\left|d f_{0}\left(\frac{\partial}{\partial x^{j}}\right)\right|^{2}=1$ and $\left|J_{j, x}(t)\right|^{2}$ achieves its maximum at $t=0$. Therefore, we have $\frac{d}{d t}\left|J_{j, x}(t)\right|^{2}=0$ and $\frac{d^{2}}{d t^{2}}\left|J_{j, x}(t)\right|^{2} \leq 0$ at $t=0$ (as one side derivative). Together with the bound on (3.3), it then implies $\dot{J}_{j, x}(0)=0$. Hence

$$
\left.\nabla_{d f_{0}\left(a_{j}\right)} V\right|_{x}=\left.\nabla_{d f_{0}\left(\frac{\partial}{\partial x^{j}}\right)} V\right|_{x}=\left.\nabla_{V} d f_{t}\left(\frac{\partial}{\partial x^{j}}\right)\right|_{t=0}=\dot{J}_{j, x}(0)=0 .
$$

This shows $\left.\nabla_{d f_{0}\left(a_{j}\right)} V\right|_{x}=0$ when $\lambda_{j}=1$.

If $\lambda_{j}<1$, condition 4 tells us $\left\langle\nabla_{d f_{0}\left(a_{i}\right)} V, d f_{0}\left(a_{j}\right)\right\rangle=0$ for $1 \leq i \leq n$. By condition 3 , we then have $\left\langle\nabla_{d f_{0}\left(a_{j}\right)} V, d f_{0}\left(a_{i}\right)\right\rangle=0$ for $1 \leq i \leq n$. Hence $\left\langle\nabla_{d f_{0}\left(a_{j}\right)} V, b_{i}\right\rangle=$ 0 if $\lambda_{i} \neq 0$. When $\lambda_{i}=0<1$, using condition 4 , one can also conclude that $\left\langle\nabla_{d f_{0}\left(a_{j}\right)} V, b_{i}\right\rangle=0$. Thus $\left\langle\nabla_{d f_{0}\left(a_{j}\right)} V, b_{i}\right\rangle=0$ for $1 \leq i \leq n$. Condition 2 then implies $\nabla_{d f_{0}\left(a_{j}\right)} V=0$.

In conclusion, we have $\nabla_{d f_{0}\left(a_{j}\right)} V=0$ for any $1 \leq j \leq n$ and $V$ is a parallel vector field. In case (ii), the variation field $V$ either vanishes on the boundary or outside a compact set of $M$. It thus implies $V=0$ on $M$. Therefore, $f_{0}=f_{1}$ and $\Sigma_{0}=\Sigma_{1}$ in case (ii). This completes the proof.

Remark 2. When $M$ is compact without boundary and $N$ has negative sectional curvature, then either $f_{0}=f_{1}$ or both $f_{0}$ and $f_{1}$ are constants. If we only know that $N$ has non-positive sectional curvature, we can still conclude that $V$ is a parallel vector field on $f_{t}(M)$ for $0 \leq t \leq 1$. The graphs of $f_{t}, 0 \leq t \leq 1$, are then minimal submanifolds of constant distance. Moreover, the Jacobi fields $\bar{J}_{i, x}(t)=d f_{t}\left(\frac{\partial}{\partial x^{i}}\right), \quad i=$ $1, \cdots, n$ are parallel along $\gamma_{x}(t)$. It implies that the induced metrics on the graphs of $f_{t}$ are the same. We also have $\dot{J}_{i, x}(t)=0$ and $\ddot{J}_{i, x}(t)=0$. The Jacobi equation thus leads to $R\left(V, d f_{t}\left(\frac{\partial}{\partial x^{i}}\right)\right) V=0$ for $1 \leq i \leq n$ and $0 \leq t \leq 1$. Hence $\langle R(V, T) V, T\rangle=0$ for any vector $T$ tangent to $f_{t}(M)$ in $N$. The results and further exploration are very similar to the case of harmonic maps as studied by Schoen and Yau in [SY].

\section{Another criterion for stability}

In this section, we will derive another criterion for the stability of minimal maps. It is in terms of bounds on the two-Jacobian $\left|\wedge^{2} d f\right|(x)$ as defined in the introduction. The theorem generalizes the results for nonparametric minimal submanifolds of codimension one.

Theorem 4.1. Let $M$ and $N$ be Riemannian manifolds with dimension $n$ and $m$ respectively, and $\Sigma$ be the graph of a map $f: M \rightarrow N$ with rank $(d f) \leq p$ for some integer $p>1$. Suppose the sectional curvature of $N$ is non-positive and $\Sigma$ is minimal in $M \times N$. Then $\Sigma$ is stable if $\left|\wedge^{2} d f\right|(x) \leq \frac{1}{p-1}$ for any $x \in M$. 
Proof. We will keep the term $\frac{1}{4} \int_{M}\left(\sum_{i, j} G^{i j} \dot{G}_{i j}\right)^{2} d v$ in the second variation formula. In the basis chosen in the proof of Lemma 3.1, we derive from (2.3)

$$
\begin{aligned}
\left.\frac{d^{2} A_{t}}{d t^{2}}\right|_{t=0}= & \int_{M}\left(\sum_{i} \frac{1}{1+\lambda_{i}^{2}}\left(\left|\nabla_{d f\left(a_{i}\right)} V\right|^{2}-\left\langle R\left(V, d f\left(a_{i}\right)\right) d f\left(a_{i}\right), V\right\rangle\right)\right. \\
& \left.-\frac{1}{2} \sum_{i, j} \frac{1}{1+\lambda_{i}^{2}} \frac{1}{1+\lambda_{j}^{2}}\left(\left\langle\nabla_{d f\left(a_{i}\right)} V, d f\left(a_{j}\right)\right\rangle+\left\langle\nabla_{d f\left(a_{j}\right)} V, d f\left(a_{i}\right)\right\rangle\right)^{2}\right) d v \\
& +\int_{M}\left(\sum_{i} \frac{1}{1+\lambda_{i}^{2}}\left\langle\nabla_{d f\left(a_{i}\right)} V, d f\left(a_{i}\right)\right\rangle\right)^{2} d v
\end{aligned}
$$

Since the sectional curvature of $N$ is non-positive, this becomes

$$
\begin{aligned}
\left.\frac{d^{2} A_{t}}{d t^{2}}\right|_{t=0} \geq & \int_{M}\left(\sum_{i} \frac{1}{1+\lambda_{i}^{2}}\left|\nabla_{d f\left(a_{i}\right)} V\right|^{2}+\left(\sum_{i} \frac{\lambda_{i}}{1+\lambda_{i}^{2}}\left\langle\nabla_{d f\left(a_{i}\right)} V, b_{i}\right\rangle\right)^{2}\right. \\
& \left.-\frac{1}{2} \sum_{i, j} \frac{1}{\left(1+\lambda_{i}^{2}\right)\left(1+\lambda_{j}^{2}\right)}\left(\lambda_{j}\left\langle\nabla_{d f\left(a_{i}\right)} V, b_{j}\right\rangle+\lambda_{i}\left\langle\nabla_{d f\left(a_{j}\right)} V, b_{i}\right\rangle\right)^{2}\right) d v \\
\geq & \int_{M}\left(\sum_{i, j} \frac{1}{1+\lambda_{i}^{2}}\left\langle\nabla_{d f\left(a_{i}\right)} V, b_{j}\right\rangle^{2}\right. \\
& +\sum_{i, j} \frac{\lambda_{i} \lambda_{j}}{\left(1+\lambda_{i}^{2}\right)\left(1+\lambda_{j}^{2}\right)}\left\langle\nabla_{d f\left(a_{i}\right)} V, b_{i}\right\rangle\left\langle\nabla_{d f\left(a_{j}\right)} V, b_{j}\right\rangle \\
& \left.-\frac{1}{2} \sum_{i, j} \frac{1}{\left(1+\lambda_{i}^{2}\right)\left(1+\lambda_{j}^{2}\right)}\left(\lambda_{j}\left\langle\nabla_{d f\left(a_{i}\right)} V, b_{j}\right\rangle+\lambda_{i}\left\langle\nabla_{d f\left(a_{j}\right)} V, b_{i}\right\rangle\right)^{2}\right) d v
\end{aligned}
$$

We break the terms into $i=j$ and $i \neq j$, and obtain

$$
\begin{aligned}
& \sum_{i, j} \frac{1}{1+\lambda_{i}^{2}}\left\langle\nabla_{d f\left(a_{i}\right)} V, b_{j}\right\rangle^{2} \\
= & \sum_{i} \frac{1}{1+\lambda_{i}^{2}}\left\langle\nabla_{d f\left(a_{i}\right)} V, b_{i}\right\rangle^{2}+\sum_{i \neq j} \frac{1}{1+\lambda_{i}^{2}}\left\langle\nabla_{d f\left(a_{i}\right)} V, b_{j}\right\rangle^{2},
\end{aligned}
$$

and

$$
\begin{aligned}
& \sum_{i, j} \frac{\lambda_{i} \lambda_{j}}{\left(1+\lambda_{i}^{2}\right)\left(1+\lambda_{j}^{2}\right)}\left\langle\nabla_{d f\left(a_{i}\right)} V, b_{i}\right\rangle\left\langle\nabla_{d f\left(a_{j}\right)} V, b_{j}\right\rangle \\
= & \sum_{i} \frac{\lambda_{i}^{2}}{\left(1+\lambda_{i}^{2}\right)^{2}}\left\langle\nabla_{d f\left(a_{i}\right)} V, b_{i}\right\rangle^{2}+\sum_{i \neq j} \frac{\lambda_{i} \lambda_{j}}{\left(1+\lambda_{i}^{2}\right)\left(1+\lambda_{j}^{2}\right)}\left\langle\nabla_{d f\left(a_{i}\right)} V, b_{i}\right\rangle\left\langle\nabla_{d f\left(a_{j}\right)} V, b_{j}\right\rangle,
\end{aligned}
$$


and

$$
\begin{aligned}
& \frac{1}{2} \sum_{i, j} \frac{1}{\left(1+\lambda_{i}^{2}\right)\left(1+\lambda_{j}^{2}\right)}\left(\lambda_{j}\left\langle\nabla_{d f\left(a_{i}\right)} V, b_{j}\right\rangle+\lambda_{i}\left\langle\nabla_{d f\left(a_{j}\right)} V, b_{i}\right\rangle\right)^{2} \\
= & \sum_{i} \frac{2 \lambda_{i}^{2}}{\left(1+\lambda_{i}^{2}\right)^{2}}\left\langle\nabla_{d f\left(a_{i}\right)} V, b_{i}\right\rangle^{2}+\sum_{i \neq j} \frac{\lambda_{j}^{2}}{\left(1+\lambda_{i}^{2}\right)\left(1+\lambda_{j}^{2}\right)}\left\langle\nabla_{d f\left(a_{i}\right)} V, b_{j}\right\rangle^{2} \\
& \left.+\sum_{i \neq j} \frac{\lambda_{i} \lambda_{j}}{\left(1+\lambda_{i}^{2}\right)\left(1+\lambda_{j}^{2}\right)}\left\langle\nabla_{d f\left(a_{i}\right)} V, b_{j}\right\rangle\left\langle\nabla_{d f\left(a_{j}\right)} V, b_{i}\right\rangle\right) .
\end{aligned}
$$

Plug these expressions into (4.1), and obtain

$$
\begin{aligned}
\left.\frac{d^{2} A_{t}}{d t^{2}}\right|_{t=0} \geq & \int_{M}\left(\sum_{i} \frac{1}{\left(1+\lambda_{i}^{2}\right)^{2}}\left\langle\nabla_{d f\left(a_{i}\right)} V, b_{i}\right\rangle^{2}\right. \\
& \left.+\sum_{i \neq j} \frac{\lambda_{i} \lambda_{j}}{\left(1+\lambda_{i}^{2}\right)\left(1+\lambda_{j}^{2}\right)}\left\langle\nabla_{d f\left(a_{i}\right)} V, b_{i}\right\rangle\left\langle\nabla_{d f\left(a_{j}\right)} V, b_{j}\right\rangle\right) d v \\
& +\int_{M}\left(\sum_{i \neq j} \frac{1}{\left(1+\lambda_{i}^{2}\right)\left(1+\lambda_{j}^{2}\right)}\left\langle\nabla_{d f\left(a_{i}\right)} V, b_{j}\right\rangle^{2}\right. \\
& \left.-\sum_{i \neq j} \frac{\lambda_{i} \lambda_{j}}{\left(1+\lambda_{i}^{2}\right)\left(1+\lambda_{j}^{2}\right)}\left\langle\nabla_{d f\left(a_{i}\right)} V, b_{j}\right\rangle\left\langle\nabla_{d f\left(a_{j}\right)} V, b_{i}\right\rangle\right) d v .
\end{aligned}
$$

The sum of the first two integrands on the right hand side of (4.2) is no less than

$$
\begin{aligned}
& \sum_{\lambda_{i} \neq 0} \frac{\left\langle\nabla_{d f\left(a_{i}\right)} V, b_{i}\right\rangle^{2}}{\left(1+\lambda_{i}^{2}\right)^{2}}+\sum_{i \neq j, \lambda_{i} \neq 0, \lambda_{j} \neq 0} \frac{\lambda_{i} \lambda_{j}\left\langle\nabla_{d f\left(a_{i}\right)} V, b_{i}\right\rangle\left\langle\nabla_{d f\left(a_{j}\right)} V, b_{j}\right\rangle}{\left(1+\lambda_{i}^{2}\right)\left(1+\lambda_{j}^{2}\right)} \\
\geq & \sum_{i \neq j, \lambda_{i} \neq 0, \lambda_{j} \neq 0} \frac{\left\langle\nabla_{d f\left(a_{i}\right)} V, b_{i}\right\rangle^{2}}{(p-1)\left(1+\lambda_{i}^{2}\right)^{2}}+\frac{\lambda_{i} \lambda_{j}\left\langle\nabla_{d f\left(a_{i}\right)} V, b_{i}\right\rangle\left\langle\nabla_{d f\left(a_{j}\right)} V, b_{j}\right\rangle}{\left(1+\lambda_{i}^{2}\right)\left(1+\lambda_{j}^{2}\right)} \\
\geq & \sum_{i \neq j, \lambda_{i} \neq 0, \lambda_{j} \neq 0} \frac{\left\langle\nabla_{d f\left(a_{i}\right)} V, b_{i}\right\rangle^{2}}{(p-1)\left(1+\lambda_{i}^{2}\right)^{2}}-\frac{\left|\left\langle\nabla_{d f\left(a_{i}\right)} V, b_{i}\right\rangle\right|\left|\left\langle\nabla_{d f\left(a_{j}\right)} V, b_{j}\right\rangle\right|}{(p-1)\left(1+\lambda_{i}^{2}\right)\left(1+\lambda_{j}^{2}\right)} \\
= & \frac{1}{p-1} \sum_{i<j, \lambda_{i} \neq 0, \lambda_{j} \neq 0} \frac{\left\langle\nabla_{d f\left(a_{i}\right)} V, b_{i}\right\rangle^{2}}{\left(1+\lambda_{i}^{2}\right)^{2}} \\
- & 2 \frac{\left|\left\langle\nabla_{d f\left(a_{i}\right)} V, b_{i}\right\rangle\right|\left|\left\langle\nabla_{d f\left(a_{j}\right)} V, b_{j}\right\rangle\right|}{\left(1+\lambda_{i}^{2}\right)\left(1+\lambda_{j}^{2}\right)}+\frac{\left\langle\nabla_{d f\left(a_{j}\right)} V, b_{j}\right\rangle^{2}}{\left(1+\lambda_{j}^{2}\right)^{2}} \\
= & \frac{1}{p-1} \sum_{i<j, \lambda_{i} \neq 0, \lambda_{j} \neq 0}\left(\frac{\left|\left\langle\nabla_{d f\left(a_{i}\right)} V, b_{i}\right\rangle\right|}{1+\lambda_{i}^{2}}-\frac{\left|\left\langle\nabla_{d f\left(a_{j}\right)} V, b_{j}\right\rangle\right|}{1+\lambda_{j}^{2}}\right)^{2} .
\end{aligned}
$$

We use $\lambda_{i} \lambda_{j} \leq \frac{1}{p-1}$ for $i \neq j$ in the second inequality. This fact follows from the condition $\left|\wedge^{2} d f\right|(x) \leq \frac{1}{p-1}$.

The sum of the last two integrands on the right hand side of (4.2), after symmetrizing the indexes, can be written as

$$
\sum_{i \neq j} \frac{\left\langle\nabla_{d f\left(a_{i}\right)} V, b_{j}\right\rangle^{2}-2 \lambda_{i} \lambda_{j}\left\langle\nabla_{d f\left(a_{i}\right)} V, b_{j}\right\rangle\left\langle\nabla_{d f\left(a_{j}\right)} V, b_{i}\right\rangle+\left\langle\nabla_{d f\left(a_{j}\right)} V, b_{i}\right\rangle^{2}}{2\left(1+\lambda_{i}^{2}\right)\left(1+\lambda_{j}^{2}\right)} .
$$


It is clearly non-negative since $\lambda_{i} \lambda_{j} \leq \frac{1}{p-1} \leq 1$ for $i \neq j$. Hence we have $\left.\frac{d^{2} A_{t}}{d t^{2}}\right|_{t=0} \geq 0$ and the minimal submanifold is stable as claimed.

\section{Acknowledgements}

The second author is grateful to Ben Andrews and Brian White for inspiring discussions. He is partially supported by an NSF grant and a Sloan fellowship. The first author is partially supported by an NSC grant in Taiwan.

\section{References}

[LO] H. B. Lawson and R. Osserman, Non-existence, non-uniqueness and irregularity of solutions to the minimal surface system. Acta Math. 139(1977), no. 1-2, 1-17.

[LW] Y.-I. Lee and M.-T. Wang, A stability criterion for nonparametric minimal submanifolds, Manuscripta Math. 112 (2003), no. 2, 161-169.

[ME] W. H., III Meeks, Uniqueness theorems for minimal surfaces. Illinois J. Math. 25 (1981), no. $2,318-336$.

[MT] M. Morse and C. Tompkins, The existence of minimal surfaces of general critical types. Ann. of Math. (2) 40 (1939), no. 2, 443-472.

[OS] R. Osserman, Minimal varieties. Bull. Amer. Math. Soc. 75 (1969), 1092-1120.

[SY] R. Schoen and S.-T. Yau, Compact group actions and the topology of manifolds with nonpositive curvature. Topology 18 (1979), 361-380.

[SH] M. Shiffman, The Plateau problem for non-relative minima. Ann. of Math. 40 (1939), 834-854.

[WA1] M.-T. Wang, The Dirichlet problem of the minimal surface system in arbitrary codimension, Comm. Pure Appl. Math. 57 (2004), no. 2, 267-281.

Department of Mathematics, National Taiwan University, Taipei 10617, Taiwan

E-mail address: yilee@math.ntu.edu.tw

Department of Mathematics, Columbia University, New York, NY 10027,USA

E-mail address: mtwang@math.columbia.edu 